\title{
A model of particles movement on a discrete contour
}

\author{
Alexander Tatashev ${ }^{1,2}$, Marina Yashina ${ }^{1,2}$ \\ ${ }^{1}$ Moscow Automobile and Road Construction State Technical University (MADI) \\ 64, Leningradsky prospect, Moscow, Russia \\ ${ }^{2}$ Moscow Technical University of Communications and Informatics (MTUCI) \\ 8-a, Aviamotornaya street, Moscow, Russia
}

\section{Article Info}

Received Dec $31^{\text {th }}, 2018$

\section{Keyword:}

Exclusive processes

Markov chains

Time-reversibility

Discrete dynamical systems

Traffic models.

\begin{abstract}
We study an exclusive process on a circle. In this paper, we study a discrete closed contour, containing $\boldsymbol{N}$ cells and $\boldsymbol{M}$ particles. Each particle occupies a cell at every time. There is not more than one particle in each cell at every moment. At every time $\boldsymbol{t}=\mathbf{0}, \mathbf{1}, \mathbf{2}, \ldots$, each particle tries to move onto a cell forward with probability $\boldsymbol{p}$, this particle tries to move back with probability $\boldsymbol{q}$, and the particle does not try to move with probability $s, p+q+s=1$. Under assumptions that $\boldsymbol{q}=\mathbf{0}$, the system of this type was considered by M. Kanai et. al. As it follows from results of these authors, in the case $\boldsymbol{q}=\mathbf{0}$, the process is time reversible, i.e., in the stationary state, the behavior of process does not change if the direction of time-axis is changed. The ergodic properties of some more general exclusive process were studied by M. Blank but, in the case $0<p, q<1$, values of steady probabilities have not been found. Under the assumptions that $M=2, N=2, s=0$, we have obtained a formula for the average velocity of particles and the particle transitions intensity. In this paper, under assumptions that $\boldsymbol{M}=2$, it has been proved the following. The process is timereversible if $\boldsymbol{M = 2 , N}$ is even and $\boldsymbol{s}=\mathbf{0}$. The process is not time-reversible if $\boldsymbol{M}=\mathbf{2}, \boldsymbol{s}>\mathbf{0}$, or $\boldsymbol{N}$ is odd and $\boldsymbol{s}=\mathbf{0}$. We have proved that the process can be nonreversible if $\boldsymbol{M} \geq 3, \boldsymbol{s}=\mathbf{0}$.
\end{abstract}

\section{Corresponding Author:}

\author{
Alexander Tatashev, \\ Moscow Automobile and Road Construction State Technical University (MADI), \\ 64, Leningradsky prospect, Moscow, Russia \\ Email: a-tatashev@yandex.ru
}

\section{Introduction}

In [1] (Belyaev, 1969) a stochastic dynamical system was introduced. This system is a traffic model on an infinite one-dimensional lattice. Any particle in this system can move in two directions. Numbers of cells are $0, \pm 1,2 \pm, \ldots$ The minimum permissible distance between two neighboring particles equals $\tau$, where $\tau$ is an odd number. At any time $0,1,2, \ldots$ with probability $r$, the particle, located in the cell $i$, moves to the right, i.e. to the cell $i+1$. With probability $l$, the particle tries to move to the left, i.e. to the cell $i-1, r+l=1$ The neighboring particle, located on the right, is a priority particle, i.e., if, at time $t$, the particle, located on the right, moves to the left, and the distance between two particles, becomes to equal $\tau-1$, then, at time $t+1$, the particle, located on the left, moves to the left onto a cell, and the distance between the particles is equal to $\tau$. It was proved that the stationary distribution of the distance between two particles is geometrical distribution with parameter depending on the flow density. It is proved that the distance between neighboring particles is a Markov chain. This chain is distributed geometrically. The expectation of this distribution corresponds to the flow density. 
A generalization of system is studied in [2] (Zele, 1972), where it is assumed that probabilities of particle transitions to the right or to the left depends on the distance between the given particles. Stationary state probabilities of the Markov chain have been found.

A formula for the average velocity of particles on infinite one-dimensional lattice has been obtained in [3] (Schadschneider, Schreckenberg, 1996). In this system particles move in accordance with the following rule. At any discrete moment, any particle tries to move onto a particle forward with probability $p$. The attempt of the particle to move is realized if the cell ahead of the particle is vacant.

In [4] (Gray, Griffeath, 2001), analytical results have been obtained for more general traffic model. In this model, a particle moves from the cell $i$ to a vacant cell $i+1$ behead of particle with probability depending on states of cells $i-1, i+2$ (cells are numbered in the direction of movement). Formulas for average velocities of particles have been found for some particular cases.

In [5] (Kanai, Nishinari, Tokihiro, 2009), a formula has been obtained for a stochastic version of the traffic model. In this system, at every step, each particle moves onto a cell forward if the cell ahead is vacant. Another form of this formula has been obtained in [6] (Buslaev, Tatashev, 2011). In [7] (Kozlov, Buslaev, Tatashev, 2013), a formula for the average velocity of particles has been obtained for a system some more general system such that, in this system, the probability of a particle transition depends on the type of the particle.

A dynamical system has been studied in [8] (Blank, 2010). In general case, the state space of the system, studied in [8], is continuous. In a particular cases, the system is equivalent to the discrete systems that is considered in [4]. In the system, studied in [8], particles moves on a straight line, which is infinite in two directions. Particles are balls of radius $r$. The minimum distance between centers of two neighboring particles is equal to $2 r$. At every discrete moment each particle tries to move on a random value $V_{i}(t)$ which is called the local velocity of the particle. In general case, the value $V_{i}$ can be negative, i.e., particles can move in both directions. Ergodic properties of stochastic versions of models, considered in [8], and its generalization were studied in [9] (Blank, 2012).

In [10] (Buslaev, Prikhodko, Tatashev, Yashina, 2005), it has been developed the following approach to model traffic flow. In accordance with this approach, the velocity of a car is the sum of a deterministic component and a stochastic component: $v=v_{d e t}+v_{s t}$. A field of cells move with velocity $v_{d e t}$. Cars are modeled by particles that occupy cells. Individual maneuvers of cars are modeled by transitions of particles to other cells.

The concept of a contour network (Buslaev contour networks) has been introduced by A.P. Buslaev, [11] (Kozlov, Buslaev, Tatashev, 2013), (Buslaev contour networks). In papers A.P. Buslaev, basic approaches for the study of complex networks. In accordance with these approaches, models are dynamical systems. The supporters of these systems are contours systems with network structures. Particles (clusters) move on contours in accordance with some rules. Limitations on a system allow to study the system analytically.

One of contour network called chainmail was studied in [11], [12], (Buslaev, Tatashev, Yashina, 2013). In this dynamical system, any particles move on a contour. The supporter of the chainmail form a twodimensional structure of contours. Any contour has common points with each of four neighboring contours. These common points are called nodes. Particles of neighboring contours cannot move through the same node simultaneously. Particles move along contours in accordance with deterministic rules. Conflict resolution rules in nodes can be stochastic. In general case, the system is not ergodic. The character of system behavior in the stationary state depends on the initial state and realization of the process.

In this paper we study a closed lattice containing $N$ cells and $M$ particles. At any discrete moment, each particle tries to move forward, tries to move back or does not try to move with probabilities $p, q, s$ respectively. If a particle tries to a cell that is occupied, then the attempt of the particle is not realized. In the case of $q=0$ (particles cannot move back) formulas for the average velocity of particles have been obtained in [5], [6]. It follows from results, obtained in [5], [6], that the system is time-reversible in the case of $q=0$. We have proved that, if $M=2$ and $N$ is even, then a unique communicating class is formed be states such that the number of even number of cells between two particles is odd. We have obtained a formula for the average velocity of particles under assumptions that $M=2, N$ is even, and $s=0$. We study time reversibility of the system. If we use the deterministic-stochastic approach, [10], then movement of a particle in backward direction can be interpreted as the movement of the car, corresponding to this particle, with the velocity which is less than the average velocity of the traffic flow. 
In Section 2, we describe the system. In Section 3, we define concepts of average velocity of particles, transitions intensity, and particles flow density. In Section 4, we give formulas for the case $q=0$ (particles cannot move back). In Section 5 formulas for stationary state probabilities, the average velocity, and transitions intensity have been obtained under the condition $M=2, s=0$, and $N$ is even. In Section 6, we study time-reversibility of the process.

\section{Description of dynamical system}

Suppose a dynamical system contains $N$ cells and $M<N$ particles, Fig. 1 . At every moment $\mathrm{t}=0,1,2, \ldots$, each particle is located in one of cells. There is no more than one particle in an any cell simultaneously. The cell $j$ is located between cells $j-1$ and $j+1$ (subtraction and addition by modulo $N$ ), $j=0,1, \ldots, N-1$. The particle $i$ is located between particles $i-1$ and $i+1$ (subtraction and addition by modulo $M$ ), $i=0,1, \ldots, M-1$. Assume that, at time $t$, a particle $i$ is in the cell $j, i=0,1, \ldots, M-1 . j=0,1, \ldots, N-1$. Then, at time $t$, with probability $p$ the particle $i$ tries to come to the cell $j+1$ (addition by modulo $N$ ). If at time $t$ the cell $j+l$ is vacant and the particle $i+1$ does not try to move to the cell $j+1$, then the attempt of the particle $i$ is realized, and this particle will be in the cell $j+1$ at time $t+1$. If the cell $j+1$ is occupied, then the particle $i$ does not move at time $t$, and, at time $t+1$, the particle $i$ will be in the cell $j$. With probability $q$ the particle $i$ tries to come to the cell $j-1$. If the cell $j-1$ is vacant at time t and the particle $i-1$ does not try to move to the cell $j-1$, then the attempt of the particle $i$ is realized, and this particle will be in the cell $j-1$. If the cell $i-1$ is occupied, then the particle $i$ does not move at time $t$. With probability s the particle $i$ does not try to move and will be in the cell $j$ at time $t+1$, Fig. 2 . If, at time $t$, the particle $i$ is in the cell $j-1$ and the particle $i+1$ is in the cell $j+1$, in the cell $j, i=0,1, \ldots, M-1$, $j=0,1, \ldots, N-1$, then a conflict occurs. In this case, no particle moves at time $t$ (strong normalization in terms $[8])$.

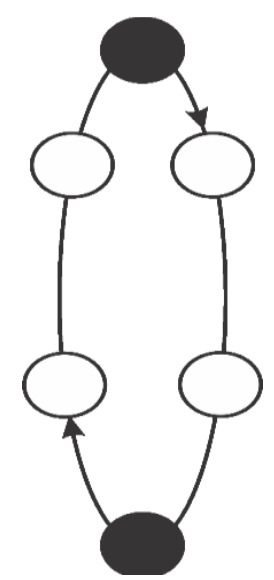

Fig. 1: A contours system, $N=6, M=2$

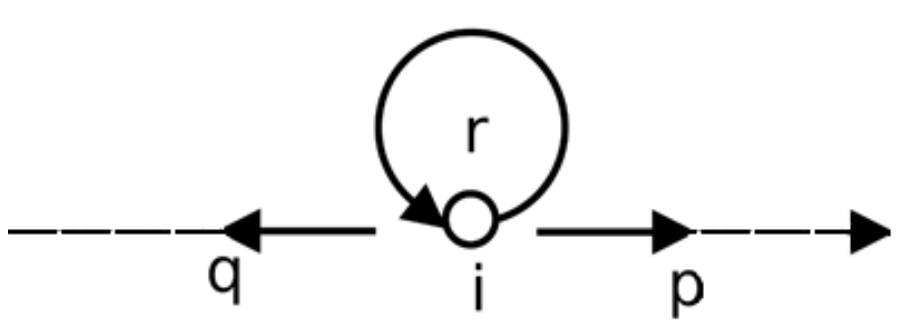

Fig. 2: Rules of movement

The state of the system is characterized by the vector $\left(d_{0}, d_{1}, \ldots, d_{N-1}\right)$, where $d_{i}=0$ if the cell $i$ is vacant, and $d_{i}=1$ if the cell $i$ is occupied, $i=0,1, \ldots, N-1$.

The initial state of the system is given. 


\section{Concepts of velocity, intensity of transitions, and flow density}

Way say that a forward transition of particle $i$ occurs if the particle $i$ moves from the cell $j$ to the cell $j+1$ (addition by modulo $N$ ), $j=0,1, \ldots, N-1$.

Way say that a back transition of the particle $i$ occurs if a particle moves from the cell $j$ to the cell $j-1$ (addition by modulo $N$ ), $j=0,1, \ldots, N-1$.

Denote by $\mathrm{S}^{+}(\mathrm{T})$ the expectation of total number of forward transitions in the time segment $[0, T-1]$; Denote by $\mathrm{S}^{-}(\mathrm{T})$ the expectation of the total number of the back transitions in the time segment $[0, T-1]$. Assume that

$$
S(T)=S^{+}(T)+S^{-}(T)
$$

The limit

$$
v=\lim _{t \rightarrow \infty} \frac{S^{+}(T)-S^{-}(T)}{T M}
$$

is called the average velocity of particles if this limit exists.

The limit

$$
Q=\lim _{t \rightarrow \infty} \frac{S(T)}{T N}
$$

is called the transitions intensity if this limit exists.

The value

is called the particles flow density.

$$
\rho=\frac{M}{N}
$$

We introduce the stochastic process $X=\left(X_{1}(t), \ldots, X_{M}(t)\right)$, where $X_{i}(t)$ is the number of vacant cells between particles $i-1$ and $i$ (subtraction by modulo $N$ ) at time $t, X_{i}(t) \geq 0, i=1, \ldots, M, X_{1}(t)+\cdots+X_{M}(t)=$ $N-M, t=0,1,2, \ldots$, The stochastic process $X$ is a Markov chain.

Denote by $v_{i}^{+}\left(X_{0}, X_{1}, \ldots, X_{M-1}\right)$ the probability that, at time $t$; a forward transition of the particle $i$ occurs provided the system is in the state $\left(X_{0}, X_{1}, \ldots, X_{M-1}\right)$ at time $t$.

Denote by $v_{i}^{-}\left(X_{0}, X_{1}, \ldots, X_{M-1}\right)$ the probability that, at time $t$, a back transition of the particle $i$ occurs provided the system is in the state

at the time $t$.

$$
\left(X_{0}, X_{1}, \ldots, X_{M-1}\right)
$$

Suppose

$$
\begin{aligned}
& v_{i}\left(X_{0}, X_{1}, \ldots, X_{M-1}\right)=v_{i}^{+}\left(X_{0}, X_{1}, \ldots, X_{M-1}\right)-v_{i}^{-}\left(X_{0}, X_{1}, \ldots, X_{M-1}\right), \\
& Q_{i}\left(X_{0}, X_{1}, \ldots, X_{M-1}\right)=v_{i}^{+}\left(X_{0}, X_{1}, \ldots, X_{M-1}\right)+v_{i}^{-}\left(X_{0}, X_{1}, \ldots, X_{M-1}\right) .
\end{aligned}
$$

The average velocity of the particle $i$ is equal to

The transitions intensity of the particle $i$ is equal to

$$
v_{i}=\sum p\left(X_{0}, X_{1}, \ldots, X_{M-1}\right) v_{i}\left(X_{0}, X_{1}, \ldots, X_{M-1}\right) .
$$

It is evident that

$$
Q_{i}=\sum p\left(X_{0}, X_{1}, \ldots, X_{M-1}\right) Q_{i}\left(X_{0}, X_{1}, \ldots, X_{M-1}\right) .
$$

Suppose

$$
\begin{gathered}
v_{o}=v_{1}=\cdots=v_{M-1} \\
Q_{o}=Q_{1}=\cdots=Q_{M-1}
\end{gathered}
$$

$$
\begin{gathered}
v=v_{i}, i=0,1, \ldots, M \\
v=v_{i}, i=0,1, \ldots, M-1,
\end{gathered}
$$




$$
Q=Q_{i}, i=0,1, \ldots, M-1 .
$$

\section{Case of probability $q=0$}

In [6], the following formula for the average velocity of particles has been obtained under the assumptions that $q=0$

$$
v=\frac{N}{M} \sum_{k=1}^{\min (M, N-M)} \frac{C p}{(1-p)^{k-1}} \cdot C_{M-1}^{k-1} C_{N-M-1}^{k-1},
$$

where

$$
\begin{gathered}
C=\left(\sum_{k=1}^{\min (M, N-M)} \frac{N}{k} \cdot C_{M-1}^{k-1} C_{N-M-1}^{k-1} \frac{1}{(1-p)^{k-1}}\right)^{-1} ; \\
C_{N}^{M}=\frac{N !}{M !(N-M) !} .
\end{gathered}
$$

In [5], a formula for the same the average velocity of particles has been also obtained under the assumptions that $q=0$. However the form of this formula differs from the form (1).

\section{Exact solution for the case of $M=2$ and even $N$}

Assume that $M=2$ and $N$ is even, $N \geq 6$.

Denote by $P_{i}$ the stationary probability of the state $\left(X_{1}=N-2-i, X_{2}=i\right)$ :

$P_{i}=P(N-2-i, i), i=0,1, \ldots, N-2$.

Theorem 1. Suppose number of particles $M=2$, the number $N \geq 6$ is even, $s=0,0<p<1$; then the following is true.

(1) There exists the stationary distribution of state probabilities/

$$
P_{i}=\left\{\begin{array}{l}
0, i=0,2, \ldots, N-2, \\
\frac{2}{N-2}, i=1,3, \ldots, N-3 .
\end{array}\right.
$$

(2) The average velocity of particles is equal to

$$
v=p-q .
$$

(3) The transitions intensity of a particle is equal to

$$
Q=1-\frac{4 p q}{N-2}
$$

Proof. If $0<p<1, i=0,1, \ldots, M-1$, then the stochastic process $X$ is a Markov chain, and the space state of this process contains a unique aperiodic communicating class. Therefore the stationary distribution of stationary states probabilities.

The stationary probabilities satisfy equations

$$
\begin{gathered}
P_{0}=p q P_{0}+p q P_{2}, \\
P_{1}=\left(p^{2}+q^{2}\right) P_{0}+\left(p^{2}+p q+q^{2}\right) P_{1}+p q P_{3}, \\
P_{i}=p q P_{i-2}+\left(p^{2}+q^{2}\right) P_{i}+p q P_{i+2}, \quad i=2,3, \ldots, N-4 \\
P_{N-3}=p q P_{N-5}+\left(p^{2}+p q+q^{2}\right) P_{N-3}+\left(p^{2}+q\right) P_{N-2},
\end{gathered}
$$




$$
\begin{aligned}
& P_{N-2}=p q P_{N-2}+p q P_{N-4}, \\
& P_{0}+P_{1}+\ldots+P_{N-2}=1 .
\end{aligned}
$$

The communicating class contains the states $P_{1}, P_{3}, \ldots, P_{N-3}$. The stationary probabilities of all these states are equiprobable. From this, the first statement of Theorem 1 follows.

The solution of the system (5)-(10) is (2).

The average velocity of particles is equal to

$v=\left(P_{3}+P_{5}+\ldots+P_{N-5}\right)(p-q)+P_{1} P_{N-3}\left(p^{2}-q^{2}\right)=$

$=\left(P_{3}+P_{5}+\ldots+P_{N-5}\right)(p-q)+\left(P_{1}+P_{N-3}\right)(p-q)(p+q)=p-q$.

The transitions intensity of a particle is equal to

$$
Q=1-\left(P_{1}+P_{N-3}\right) p q
$$

From (5)-(11), the formula (3) follows. From (5)-(10), (12), the formulas (4), (5) follows. Theorem 1 has been proved.

Remark 1. If $p \rightarrow 1$; then, in accordance with (3), $v \rightarrow 1$. This consists with results obtained in [2, 4], where the system was studied under assumption that $p=1$.

\section{Reversibility of the system}

\subsection{Formulation of problem}

Suppose

$$
p_{i j}=P(X(t)+1=j \mid P(X(t))=i)
$$

$p_{i j}$ is the probability of the transition from the state $i$ to the state $j$;

$$
\bar{p}_{i j}=P(X(t-1)=j \mid P(X(t)=i)) ;
$$

provided the system in the stationary state.

In accordance with a definition given in [13], a stochastic process is called reversible if and only if for any $i, j$ such that $p_{j}>0$ the equality

holds.

$$
\tilde{p}_{i j}=p_{i j}
$$

The equality (13) holds if and only if the equality

holds.

$$
p_{j} p_{j i}=p_{i} p_{i j}
$$

We study reversibility of the process $X(t)$.

\subsection{Reversibility of the process $X(t)$ in the case of $q=0$}

Assume that $q=0$, i.e., particles move only in one direction. In this case, in accordance with results $[6,7]$, the process $X(t)$ is reversible.

\subsection{Reversibility of the process $X(t)$ in the case of $M=2$, even $\mathrm{N}$, and $s=0$}

In Subsection 6.3, we assume that $M=2, N$ is even, and $s=0$.

Theorem 2. Suppose number of particles $M=2$, the number $N \geq 6$ is even, $s=0,0<p<1$; then the process $X(t)$ is reversible.

Proof. Under conditions of the theorem, we have that $p_{i}>0$ only for odd numbers, and

$$
p_{i, i+2}=p q, i=1,3, \ldots, N-5
$$




$$
\begin{gathered}
p_{i, i-2}=p q, i=3,5, \ldots, N-3 \\
p_{i j}=0, i=1,3, \ldots, N-3, j \neq i, i-2, i+2 .
\end{gathered}
$$

From this and Theorem 1, it follows that (14) holds, and thus Theorem 2 has been proved.

\subsection{Non-reversibility of the process $X(t)$ in the case of $M=2$, even $N$ and $s=0$}

In Subsection 6.4, we consider the system under assumptions that $M=2, N$ is odd, and $s=0$.

Theorem 3. Suppose number of particles $M=2$, the number $N \geq 5$ is odd, $s=0,0<p<1$; then the process $X(t)$ is not reversible.

Proof. Under the conditions of the theorem, we have that all states of the system form a unique aperiodic communicating class. If the process $X(t)$ is reversible, then the stationary probabilities satisfy (14) (17). But in the case of odd $N$, all probabilities are positive and cannot satisfy (14) - (17). This contradiction proves Theorem 3.

\subsection{Non-reversibility of the process $X(t)$ in the case of $M=2$ and $s>0$}

In Subsection 6.5, we consider the system under assumptions that $M=2, s<1$.

Theorem 4. Suppose number of particles $M=2,0<s<1$, then the process $X(t)$ is not reversible.

Proof. Under the conditions of the theorem, we have that all states of the system form a unique aperiodic communicating class. Stationary probabilities of all states are positive. We have.

$$
\begin{aligned}
& p_{01}=p^{2}+q^{2}+p s+q s, \\
& p_{10}=p_{12}=p_{21}=p s+q s, \\
& p_{02}=p_{20}=p q \\
& p_{12}=p s+q s \\
& p_{21}=p s+q s .
\end{aligned}
$$

Assume that the process $X(T)$ is reversible. Using (13), (18)-(22), we get

$$
\begin{aligned}
& P_{1}=P_{0} \cdot \frac{p_{01}}{p_{10}}=P_{0} \cdot \frac{p^{2}+q^{2}+p s+q s}{p s+q s}, \\
& P_{2}=P_{0} \cdot \frac{p_{12}}{p_{21}}=P_{0} \cdot \frac{p s+q s}{p s+q s}=P_{0}, \\
& P_{2}=P_{1} \cdot \frac{p s+q s}{p s+q s}=P_{1},
\end{aligned}
$$

Using (23)-(25), we get

$$
\left(p^{2}+q^{2}+p s+q s\right) /(p s+q s)=1 .
$$

But (26) does not hold for $0<p$; $q$; $s<1$ : This contradiction proves Theorem 4 . 


\subsection{Non-reversibility in the case $s=0$ and $M=3$}

The following example shows that the process can be non-reversible in the case $\mathrm{s}=0$ and $M>2$.

Suppose $N=6, M=3, p=q=1 / 2, s=0$. We shall define the process $Y(t)$ as follows. There are three states of this process. Assume that $Y(t)=1$ if all particles form one cluster, i.e., three particles occupy neighboring cells; $Y(t)=2$ if two particles form a cluster and one particle is not in this cluster; $Y(t)=3$ if there is a vacant cell between any two particles. Thus $Y(t)$ is the number of clusters at time $t$. All states of the process $Y(t)$ is the number of clusters at time $t$. All states of the process $Y(t)$ form a unique class of communicating states. Denote by $P_{i}$ the stationary probability of the state $Y=i, i=1,2,3$.

Stationary probabilities of states satisfy the system of equations

$$
\begin{aligned}
& P_{1}=\frac{P 1}{4}+\frac{3 P 2}{16}+\frac{3 P 3,}{8} \\
& P_{2}=\frac{P 1}{2}+\frac{11 P 2}{16}+\frac{3 P 3}{8}, \\
& P_{3}=\frac{P 1}{4}+\frac{P 2}{8}+\frac{P 3}{4}, \\
& P_{1}+P_{2}+P_{3}=1 .
\end{aligned}
$$

The solution of this system of equations

We have

$$
P_{1}=\frac{4}{17}, P_{2}=\frac{10}{17}, P_{3}=\frac{3 .}{17}
$$

$$
P_{1} p_{12}=\frac{4}{17} \cdot \frac{1}{2} \neq \frac{10}{17} \cdot \frac{3}{16}=P_{2} p_{21}
$$

Thus the process is not reversible.

\section{Conclusion}

We have obtained formulas for average velocity of particles and transition intensity under the assumptions that the number of particles equals 2 , the number of cells is even, and the probability that the particle does not try to move equals 0 . We study the reversibility of the system processing. It is known that, if particles move only in one direction, the process is time-reversible. Under assumptions that the number of particles equals $2,0<p<1,0<q<1$, we have proved the following. The process is time-reversible if the number of cells is even, and the probability that the particle does not move is equal to 0 . The process is time-reversible if only one of these two conditions holds.

\section{ACKNOWLEDGEMENTS}

This work has been supported by the Russian Foundation for Basic Research, Grant No. 17-01-00821-a and Grant No. 17-07-01358-a.

\section{REFERENCES}

[1] Belyaev Yu.K. About simplified model of movement without overtaking. Izvestiya AN SSSR. Tekhnicheskaya kibernetika, 1972, no. 5, pp. 100 - 103. (In Russian.)

[2] Zele U. Generalization of model of movement without overtaking. Izvestiya AN SSSR. Tekhnicheskaya kibernetika, 1972, no. 5, pp. 100 - 103. (In Russian.)

[3] Schadschneider A., Schreckenberg M. Car-oriental mean-field theory for traffic theory. 1996. arXiv:cond-mat/9612113v1 
[4] Gray L., Griffeath D. The ergodic theory of traffic jams. J. Stat. Phys., 2001, vol. 105, no's 3/4, pp. 413-452.

[5] Kanai M., Nishinari K., Tokihiro T. Exact solution and asymptotic behaviour of the asymmetric simple exclusion process on a ring. 18 May 2009. arXiv.0905.2795v1 [cond-mat-stat-mech]

[6] Buslaev A.P., Tatashev A.G. Particles flow on the regular polygon. Journal of Concrete and Applicable Mathematics, 2011, vol. 9, no. 4, pp. 290 - 303.

[7] Kozlov V.V., Buslaev A.P., Tatashev A.G. Monotonic random walks and clusters flows on networks. Models and applications, Lambert Academician Publishing, Saarbrucken, Germany, 2013, no. 78724.

[8] Blank M. Metric properties of discrete time exclusion type processes in continuum. J. Stat. Phys. 2010, vol. 140 , pp. $170-197$.

[9] Blank M. Stochastic stability of traffic maps. arXiv:1210.2236v1 [math.DS] 8 Oct 2012

[10] Buslaev A.P., Prikhodko V.M., Tatashev A.G., Yashina M.V. The deterministic-stochastic flow model, 2005. arXiv:physics/0504139[physics.sos-ph], pp. $1-21$.

[11] Kozlov V.V., Buslaev A.P., Tatashev A.G. On synergy of totally connected flow on chainmails (CMSSE-2013, Cadiz, Spain ), vol. 3, pp. $861-873$.

[12] Buslaev A.P., Tatashev A.G., Yashina M.V. Qualitative properties of dynamical system on toroidal chainmail. 11th International Conference of Numerical Analysis Mathematics 2013 (ICNAAM 2013, Rhodes, Greece, 21 - 27 September 2013. AIP Conference Proceedings 1558, pp. 1144 - 1147 (2013).

[13] Kemeny J.G., Snell J.L., Finite Markov chains. - The University Series in Undergraduate Mathematics. - Princeton: Van Nostrand, 1960. 